\title{
THE SENSITIVITY OF THE EYE TO TWO KINDS OF CONTINUOUS TRANSFORMATION OF A SHADOW-PATTERN ${ }^{1}$
}

\author{
KAI VON FIEANDT \\ University of Helsinki \\ AND JAMES J. GIBSON \\ Cornell University
}

Many years ago, the French mathematician Poincaré (1913, p. 250 ff.) put a psychological question which has not yet been answered by psychologists: How does the eye distinguish between the change of position of a thing and the change of state of a thing? By "position," he meant the distance and orientation of an object in the environment, which can change when either the observer or the object moves. By "state," he meant the identity or rigidity of an object in the environment, which can change when the object grows or changes shape (even to the extent of becoming a different object). If vision presents to the eye only a projection or silhouette of the object, how does the eye discriminate between the perspective alterations of this projection and the alterations arising from real changes of the object? For example, how can the observer tell the difference between approach and growth, that is, between the object getting nearer and the object larger? How can he tell the difference between a turning motion of the object and a deformation of the object when, in both cases, its silhouette is compressed in one dimension? On the one hand,

1 This experiment was performed at Cornell University where the first author was visiting on a grant from the U. S. Department of State. The first author designed the apparatus and carried out the experiment. The second author is responsible for the theory. We are indebted to Paul Olum of Cornell University for advice in the field of geometry. the silhouettes or visual sensations of objects are transformed when we move from one point of view to another, or when rigid objects themselves change position relative to the point of view. On the other hand, they are transformed when fluid or elastic objects change with respect to size or shape-a cloud or a surface of living tissue. But the changes of relative position are not confused in perception with the changes of structure or state. Men and animals seem to distinguish the rigid and permanent surfaces of the environment from the elastic or animate surfaces of the environment with considerable success. But why do both not look indifferently elastic since both may undergo transformation?

Poincaré, being a geometer, realized that the two kinds of transformations belong to different "groups." $\mathrm{He}$ thought that a man might learn to distinguish a change of position from a change of state by virtue of the fact that the first kind of transformation can always be reversed by a locomotor movement of the observer himself whereas the second kind of transformation cannot. That is, the observer can always move to a point of view which nullifies the first kind of transformation and re-establishes the original sensation and he can learn to associate his movement with the change of sensation. This is an interesting theory. However, a less speculative and more direct explanation is possible. It would be that an 
eye, for reasons to be investigated, is sensitive to a change of form, and, moreover, to the type of a change of form. In this respect, an eye is to be contrasted with a camera. The eyes of animals and men are very good at detecting motions; perhaps they are just as good at discriminating types of motions. It might be that the one group of continuous transformations, the perspectives of rigid objects, constitutes one kind of stimulus for vision and that the other group, the "rubbery" transformations, constitutes another kind of stimulus for vision.

This hypothesis gains plausibility from the results of an experiment by Gibson and Gibson (1957) in which it was shown that a continuous perspective transformation (with polar projection) is a sufficient stimulus for an impression of rigid motion in space. $^{2}$ The transformation was carried by a pattern of shadows on the translucent screen of a point-source shadow projector (Gibson, 1957). The apparent surface seemed to rotate in the space behind the screen - that is, to change its position without change of its structure. But the experiment did not test the prediction that a continuous transformation of the other kind would give an impression of non-rigid motion-that is, a change of structure without change of position in space. The aim of the present experiment was to compare the perceptions induced by the two kinds of transformation sequences, everything else being equal so far as possible, and to determine whether corresponding impressions of rigid and of non-rigid motion would occur spontaneously.

2 This research is allied to the stereokinetic phenomenon of Benussi and Musatti (1924) and to the kinetic depth effect of Wallach, and his collaborators (e.g., Wallach Weisz, \& Adams, 1956).

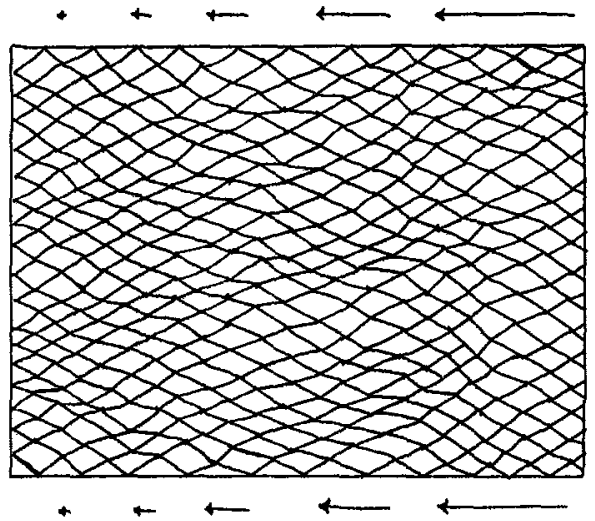

FIG. 1. The shadow-pattern. Note that the network is irregular and unfamiliar, and that a linear perspective cue is not available for the perception of slant. The change of pattern is indicated crudely by the arrows indicating displacements of elements. In the case of the perspective transformation, however, there is a vertical component of displacement as well as the horizontal component.

\section{MethoD}

Apparatus.-A point-source shadow projector is designed to produce an ongoing perspective transformation. It was necessary to modify the apparatus so that it would also produce an ongoing transformation of the "rubbery" kind, and so that it could be shifted from the first to the second kind of transformation without any realization by $O$ that the apparatus has been changed.

For a decisive test, the two kinds of motion should be superficially similar but not mathematically similar. It was decided to fill the whole rectangular screen with a pattern (an irregular network of dark lines) which could be compressed from the right to the left of the window and then decompressed to restore the original pattern. There was to be always, in short, a gradient of leftward velocities of the elements of the pattern from a maximum on the right to zero on the left and then the same gradient of rightward velocities (Fig. 1). But one kind of compression of the image was to be the projection of a rigid surface which had been made to turn, whereas the other kind was to be the projection of an elastic surface which had been made to contract.

The shadow-caster behind the screen was therefore constructed in a special way. It consisted of a net of elastic thread stretched on a rectangular frame whose edges were 
projected outside the frame of the window and were therefore never visible. The $E$ could either rotate the frame around a vertical axis at its left-hand edge or allow the right-hand edge to slide inward on a pair of guides. Both motions were silent. At the top and bottom of the frame the net was attached by rubber bands so that the sliding motion was distributed over the whole area of the net. During rotation the slide was locked in its outermost position; during contraction the frame was kept parallel to the plane of the window. ${ }^{3}$

With this arrangement, a $20^{\circ}$ rotation of the frame (toward the window) was equivalent to an $8-\mathrm{cm}$. inward displacement of the slide-equivalent with respect to the number of diamond-shaped elements of the shadows entering the window to the right. Stops were placed to limit the motions of the frame at these extremes. The $E$ could thus, at will, produce either of two types of cyclical motion of the shadow pattern, operating the frame by hand at the rate of one cycle in $4 \mathrm{sec}$. The window was $38 \times 40 \mathrm{~cm}$. at a distance of $140 \mathrm{~cm}$. from $O$ 's eye, and the point-source was also $140 \mathrm{~cm}$. behind the window. The frame was $39 \times 47 \mathrm{~cm}$. (when fully stretched) at a distance of $57 \mathrm{~cm}$, behind the window (when parallel to the window). The $O$ observed the window through a monocular aperture which hid the apparatus.

Procedure.-One way of comparing the perceptions aroused by two stimulus events is to determine whether the events are spontaneously named or identified with different words. This is not easy, however, if the ordinary language of the $O$ s does not include accepted words for the different events. Another way is to determine whether the events are distinguished or discriminated by the consistent assignment of different words to different events, even though the words do not have accepted meaning. And a third way is to determine whether the transition from the presenting of one event to the presenting of the other arouses a reaction consistent with the direction of the

${ }^{3}$ The transformation of the shadow during lateral contraction of the frame was actually an approximation to the limiting case of a perspective transformation with parallel protection instead of polar protection. That is, it was similar to (but not wholly identical with) the motion which the shadow would have undergone if the frame had been rotated with the light source at a very great distance. This is a transformation which converts a square into a rectangle. With polar projection a square is converted into a trapezoid. transition. All three methods were used in the present experiment.

A naive $O$ was brought into the dimly lighted experimental room, seated, and told:

"When you look through this tube you will see something. Please describe what you think it might be. When it begins to move, say what kind of movement it is. If the kind of movement changes, what new kind is it?"

The shadow of the net was motionless for a few seconds, and then cycles of motion were presented. Half the Os began with contraction and half with rotation. After three cycles (about $12 \mathrm{sec}$.) the other kind of motion was introduced without interruption for three cycles, and this alternation was continued until each kind of motion had been presented three times. During these six presentations $O$ was freely reporting. This was the first part of the experiment, intended to find out if the verbal descriptions fell into two categories, and whether or not these could be taken to mean rigid and non-rigid motion. No suggestions were given at any time.

Nearly all $O_{\mathrm{S}}$ (20 out of 22 tested) could be considered to have distinguished two classes of motion, although they had difficulty in finding words to describe what they saw. Using the terms spontaneously used by $O$, this group was then given the further instructions:

"From now on always use these names when you see the corresponding movements. Report whenever the movement changes from one to the other."

There followed 50 three-cycle presentations, 25 of contraction and 25 of rotation in a prearranged random order, half the $O$ s beginning with one and half with the other. There were pauses after each trial so that the verbal identification could be recorded by $E$. This was the second part of the experiment.

Finally, a continuous series of motioncycles was presented consisting of 47 transitions from contraction to rotation or the reverse, each transition being separated from the preceding by either three or four cycles of motion, these two possibilities being randomized. A record was kept of the changes reported or missed.

As an extra guarantee against the use of auditory cues for distinguishing the motions of the shadow-caster, a moderate masking noise was produced with a tape recorder placed near $O$.

Subjects.-Twenty $O_{\text {s }}$ completed the experiment, of which 18 were undergraduate students with little or no training in psychology and two were psychologists. 


\section{RESUlts}

The motionless shadow of the net was always seen as something in the frontal plane. The subsequent cycles of movement were described in a variety of terms whose meaning had to e interpreted. But the reports clearly fell into two types, and the change from one to the other movement of the shadow-caster was promptly noticed and reported.

The conclusion that the two kinds of transformation aroused two different perceptions is also warranted by the second part of the experiment. Out of 1000 judgments required of the

Os, 979 or $98 \%$ were scored as correct, in that they consistently applied the terminology developed in the first six presentations.

The conclusion that the transitions were immediately responded to is proved by the third part of the experiment. Of 940 changes from rotation to compression or the reverse, all were responded to, and only one $O$ on one occasion reported a change in the motion when none had occurred.

May we conclude that the Os spontaneously saw the same events which the $E$ s saw in response to the two kinds of transformations? These events were, on the one hand, a rigid turning movement of an apparent surface in the space behind the window, and, on the other, an elastic or squeezing movement of an apparent surface, usually without change of depth but sometimes with a vague impression of it. The rigid rotation in depth could, with a shift of attitude, be seen as a compression, but this was not the same kind of compression as the elastic movement. The experience of compression obtained with the perspective transformation was, moreover, unstable.

The Os did not use the above terms to describe the events in question. They used familiar words like "wire fencing," "swinging toward me," "back and forth," "compression," "diagonal movement," "horizontal movement," and the like.
The interpretation of such words is subject to error. Nevertheless the judgment of the authors is that $O_{s}$ in this experiment perceived essentially the same events. The perceptions differed in lacking the precision which can be achieved by using such terms as "rigid" and "elastic."

It is clear that, by two objective criteria, unsophisticated $O s$ can distinguish between a continuous perspective transformation and a nonperspective transformation similar to it with respect to the gross pattern of displacements of elements. The visual system is sensitive to different kinds of change of projected form, at least in adults. This fact would explain the puzzle of Poincare- why we do not confuse the rigid movements of surfaces in depth with the elastic movements of surfaces in the frontal plane. We cannot conclude with certainty that these $O$ s did actually perceive a rigid surface in one case and a non-rigid surface in the other, for the extent to which the abstract property of rigidity was induced in our experimental situation is not clear. Nevertheless, the words used spontaneously by the $O$ s suggest a tendency to do so.

\section{Summary}

Continuous perspective transformations of a rigid object and continuous nonperspective transformations of an elastic object were presented to $O$ s under three conditions to determine whether discrimination of these would occur. All tests supported the inference that the discrimination is very efficient.

\section{REFERENCES}

Gibson, J. J. Optical motions and transformations as stimuli for visual perception. Psychol. Rev., 1957, 64, 288-295.

Gibson, J. J., \& Gibson, E. J. Continuous perspective transformations and the perception of rigid motion. J. exp. Psychol., 1957, 54, 129-138.

Musattr, C. L. Sui fenomeni stereocinetici. Arch. Ital. Psicol., 1924, 3, 105-120.

Poncart., $\mathrm{H}$. The value of science (Trans. by G. B. Halstead), New York: Science Press, 1913.

Wallach, H., Weisz, A., \& Adams, P. A. Circles and derived figures in rotation. A mer. J. Psychol., 1956, 69, 48-59.

(Received June 27, 1958) 